\title{
Joint Discrete Approximation of Analytic Functions by Hurwitz Zeta-Functions
}

\author{
Aidas Balčiūnas ${ }^{a}$, Virginija Garbaliauskienè $^{b}$, \\ Violeta Lukšiené ${ }^{a}$, Renata Macaitiene $\dot{\mathrm{e}}^{c}$ and \\ Audronè Rimkevičienè ${ }^{c}$ \\ ${ }^{a}$ Institute of Mathematics, Faculty of Mathematics and Informatics, Vilnius \\ University \\ Naugarduko g. 24, LT-03225 Vilnius, Lithuania \\ ${ }^{b}$ Institute of Regional Development, Šauliai Academy, Vilnius University \\ P. Višinskio g. 25, LT-76351 Šiauliai, Lithuania \\ ${ }^{c}$ Faculty of Business and Technologies, Šiauliai State College \\ Aušros al. 40, LT-76241 Šiauliai, Lithuania \\ E-mail: aidas.balciunas@mif .vu.lt \\ E-mail: virginija.garbaliauskiene@sa.vu.lt \\ E-mail: violeta.franckevic@mif.vu.lt \\ E-mail(corresp.): r.macaitiene@svako.lt \\ E-mail: a.rimkeviciene@svako.lt
}

Received June 7, 2021; revised December 8, 2021; accepted December 8, 2021

\begin{abstract}
Let $H(D)$ be the space of analytic functions on the strip $D=\{\sigma+i t \in$ $\mathbb{C}: 1 / 2<\sigma<1\}$. In this paper, it is proved that there exists a closed non-empty set $F_{\alpha_{1}, \ldots, \alpha_{r}} \subset H(D)$ such that every collection of the functions $\left(f_{1}, \ldots, f_{r}\right) \in F_{\alpha_{1}, \ldots, \alpha_{r}}$ is approximated by discrete shifts $\left(\zeta\left(s+i k h_{1}, \alpha_{1}\right), \ldots, \zeta\left(s+i k h_{r}, \alpha_{r}\right)\right), h_{j}>0, j=$ $1, \ldots, r, k \in \mathbb{N} \cup\{0\}$, of Hurwitz zeta-functions with arbitrary parameters $\alpha_{1}, \ldots, \alpha_{r}$.
\end{abstract}

Keywords: Hurwitz zeta-function, space of analytic functions, weak convergence, universality.

AMS Subject Classification: 11M35.

Copyright (C) 2022 The Author(s). Published by Vilnius Gediminas Technical University This is an Open Access article distributed under the terms of the Creative Commons Attribution License (http://creativecommons.org/licenses/by/4.0/), which permits unrestricted use, distribution, and reproduction in any medium, provided the original author and source are credited. 


\section{Introduction}

Let $s=\sigma+i t$ be a complex variable, and $\alpha, 0<\alpha \leqslant 1$, be a fixed parameter. The Hurwitz zeta-function $\zeta(s, \alpha)$ is defined, for $\sigma>1$, by the Dirichlet series

$$
\zeta(s, \alpha)=\sum_{m=0}^{\infty} \frac{1}{(m+\alpha)^{s}},
$$

and can be continued analytically to the whole complex plane, except for a simple pole at the point $s=1$ with residue 1 . For $\alpha=1$, the function $\zeta(s, \alpha)$ becomes the Riemann zeta-function $\zeta(s)$, and, for $\alpha=\frac{1}{2}, \zeta\left(s, \frac{1}{2}\right)=\left(2^{s}-1\right) \zeta(s)$. Further, the function $\zeta(s, \alpha), \alpha \neq 1 ; \frac{1}{2}$, has no Euler's product, and this is reflected in its value distribution.

Suppose that $\mathfrak{a}=\left\{a_{m}: m \in \mathbb{N}_{0}=\mathbb{N} \cup\{0\}\right\}$ be a periodic sequence of complex numbers. A generalization of the function $\zeta(s, \alpha)$ is the periodic Hurwitz zeta-function

$$
\zeta(s, \alpha ; \mathfrak{a})=\sum_{m=0}^{\infty} \frac{a_{m}}{(m+\alpha)^{s}}, \quad \sigma>1,
$$

which also has the meromorphic continuation to the whole complex plane.

Analytic properties of the functions $\zeta(s, \alpha)$ and $\zeta(s, \alpha ; \mathfrak{a})$, including the approximation of analytic functions, depend on the arithmetic nature of the parameter $\alpha$. Let $D=\{s \in \mathbb{C}: 1 / 2<\sigma<1\}$. Denote by $H(D)$ the space of analytic functions on $D$ endowed with the topology of uniform convergence on compacta. Approximation of all functions of the space $H(D)$ by shifts $\zeta(s+i \tau, \alpha)$ and $\zeta(s+i \tau, \alpha ; \mathfrak{a}), \tau \in \mathbb{R}$, is called universality of the functions $\zeta(s, \alpha)$ and $\zeta(s, \alpha ; \mathfrak{a})$, respectively. More precisely, the following results are known.

Denote by $\mathcal{K}$ the class of compact subsets of the strip $D$ with connected complements, and by $H(K)$ with $K \in \mathcal{K}$ the class of continuous functions on $K$ that are analytic in the interior of $K$. Let meas $A$ stand for the Lebesgue measure of a measurable set $A \subset \mathbb{R}$. Suppose that the number $\alpha$ is transcendental or rational $\neq 1$ or $1 / 2$, and $K \in \mathcal{K}, f(s) \in H(K)$. Then, for every $\varepsilon>0$,

$$
\liminf _{T \rightarrow \infty} \frac{1}{T} \text { meas }\left\{\tau \in[0, T]: \sup _{s \in K}|\zeta(s+i \tau, \alpha)-f(s)|<\varepsilon\right\}>0 .
$$

Different proofs of the latter inequality are given in $[1,10,36]$ and [28].

The above theorem is of continuous type. Also, a similar result of discrete type is known. Denote by $\# A$ the cardinality of a set $A$, and let $N$ run over the set $\mathbb{N}_{0}$. For $\alpha$ rational $\neq 1$ or $1 / 2$, let $h>0$ be arbitrary, while, for transcendental $\alpha$, let $h$ be such that $\exp \{(2 \pi l) / h\}$ is irrational for all $l \in \mathbb{N}$. Let $K$ and $f(s)$ be as above, then, for every $\varepsilon>0$,

$$
\liminf _{N \rightarrow \infty} \frac{1}{N+1} \#\left\{0 \leqslant k \leqslant N: \sup _{s \in K}|\zeta(s+i k h, \alpha)-f(s)|<\varepsilon\right\}>0 .
$$

For the proof, see $[1,28,35]$.

Universality results for the function $\zeta(s, \alpha)$ also follows from the Mishou theorem on the joint universality of the Riemann and Hurwitz zeta-functions 
[33] and other results of a such type $[5,6,7,18,20]$. More general, shifts $\zeta(s+$ $i \varphi(k), \alpha)$ with a certain function $\varphi(k)$ were used in [19]. The shifts $\zeta\left(s+i h \gamma_{k}, \alpha\right)$, where $0<\gamma_{1}<\gamma_{2}<\cdots \leqslant \gamma_{k} \leqslant \gamma_{k+1} \leqslant \cdots$ is a sequence of imaginary parts of non-trivial zeros of the Riemann zeta-function were applied in $[3,23,26]$ and [32]. Analogical universality theorems for the function $\zeta(s, \alpha ; \mathfrak{a})$ were proved in $[11,29,31]$, and follow from joint universality theorems for periodic zetafunctions (see, for example, $[12,14,17,22,25,30]$ ).

Universality of the functions $\zeta(s, \alpha)$ and $\zeta(s, \alpha ; \mathfrak{a})$ with algebraic irrational parameter $\alpha$ is a very complicated and open problem.

In $[13,15]$, for universality of $\zeta(s, \alpha)$, the linear independence over the field of rational numbers for the sets

$$
\left\{\log (m+\alpha): m \in \mathbb{N}_{0}\right\} \text { and }\left\{\left(\log (m+\alpha): m \in \mathbb{N}_{0}\right), 2 \pi / h\right\}
$$

was required. This requirement is weaker than the transcendence of $\alpha$, however, examples of such $\alpha$ are not known. In the joint case, the above sets were generalized $[13,16]$ by

$$
\left\{\left(\log \left(m+\alpha_{1}\right): m \in \mathbb{N}_{0}\right), \ldots,\left(\log \left(m+\alpha_{1}\right): m \in \mathbb{N}_{0}\right)\right\}
$$

and

$$
\left\{\left(h_{1} \log \left(m+\alpha_{1}\right): m \in \mathbb{N}_{0}\right), \ldots,\left(h_{r} \log \left(m+\alpha_{r}\right): m \in \mathbb{N}_{0}\right), 2 \pi\right\} .
$$

There are known several results of approximation of analytic functions by shifts of the functions $\zeta(s, \alpha)$ and $\zeta(s, \alpha ; \mathfrak{a})$ with algebraic irrational parameter $\alpha$, however, the set of approximated functions is not identified. The first results of such a kind has been obtained in [2]. Suppose that $0<\alpha<1$ is arbitrary. Then there exists a closed non-empty subset $F_{\alpha} \subset H(D)$ such that, for every compact $K \subset D, f(s) \in F_{\alpha}$ and $\varepsilon>0$, inequality (1.1) holds. The analogical statements for the functions $\zeta(s, \alpha ; \mathfrak{a})$ and the Lerch zeta-function are given in [9] and [21], respectively. Generalizations of [2] for the Mishou theorem were obtained in [24]. In [8], the following joint approximation theorem for Hurwitz zeta-functions has been proved.

Theorem 1. Suppose that the numbers $0<\alpha_{j}<1, \alpha_{j} \neq 1 / 2, j=1, \ldots, r$, are arbitrary. Then there exists a closed non-empty set $F_{\alpha_{1}, \ldots, \alpha_{r}} \subset H^{r}(D)$ such that, for every compact sets $K_{1}, \ldots, K_{r} \subset D,\left(f_{1}, \ldots, f_{r}\right) \in F_{\alpha_{1}, \ldots, \alpha_{r}}$ and $\varepsilon>0$

$$
\liminf _{T \rightarrow \infty} \frac{1}{T} \operatorname{meas}\left\{\tau \in[0, T]: \sup _{1 \leqslant j \leqslant r} \sup _{s \in K_{j}}\left|\zeta\left(s+i \tau, \alpha_{j}\right)-f_{j}(s)\right|<\varepsilon\right\}>0 .
$$

Moreover, the limit

$$
\lim _{T \rightarrow \infty} \frac{1}{T} \operatorname{meas}\left\{\tau \in[0, T]: \sup _{1 \leqslant j \leqslant r} \sup _{s \in K_{j}}\left|\zeta\left(s+i \tau, \alpha_{j}\right)-f_{j}(s)\right|<\varepsilon\right\}>0
$$

exists for all but at most countably many $\varepsilon>0$.

The aim of this paper is a discrete version of Theorem 1. For brevity, let $\underline{\alpha}=\left(\alpha_{1}, \ldots, \alpha_{r}\right)$ and $\underline{h}=\left(h_{1}, \ldots, h_{r}\right)$. 
Theorem 2. Suppose that the numbers $0<\alpha_{j}<1, \alpha_{j} \neq 1 / 2$ and positive numbers $h_{j}, j=1, \ldots, r$, are arbitrary. Then there exists a closed nonempty set $F_{\underline{\alpha}, \underline{h}} \subset H^{r}(D)$ such that, for every compact sets $K_{1}, \ldots, K_{r} \subset D$, $\left(f_{1}, \ldots, f_{r}\right) \in F_{\underline{\alpha}, \underline{h}}$ and $\varepsilon>0$,

$$
\liminf _{N \rightarrow \infty} \frac{1}{N+1} \#\left\{0 \leqslant k \leqslant N: \sup _{1 \leqslant j \leqslant r} \sup _{s \in K_{j}}\left|\zeta\left(s+i k h_{j}, \alpha_{j}\right)-f_{j}(s)\right|<\varepsilon\right\}>0 .
$$

Moreover, "lim inf" can be replaced by "lim" for all but at most countably many $\varepsilon>0$.

It will be proved that the set $F_{\underline{\alpha}, \underline{h}}$ is the support of a certain $H^{r}(D)$-valued random element.

\section{Probabilistic results}

Denote by $\mathcal{B}(\mathbb{X})$ the Borel $\sigma$-field of the space $\mathbb{X}$, and, for $A \in \mathcal{B}\left(H^{r}(D)\right)$, define

$$
P_{N, \underline{\alpha}, \underline{h}}(A)=\frac{1}{N+1} \#\{0 \leqslant k \leqslant N: \underline{\zeta}(s+i k \underline{h}, \underline{\alpha}) \in A\},
$$

where

$$
\underline{\zeta}(s+i k \underline{h}, \underline{\alpha})=\left(\zeta\left(s+i k h_{1}, \alpha_{1}\right), \ldots, \zeta\left(s+i k h_{r}, \alpha_{r}\right)\right) .
$$

In this section, we deal with weak convergence of $P_{N, \underline{\alpha}, \underline{h}}$ as $N \rightarrow \infty$.

We start with definition of one probability space. Define

$$
\Omega=\prod_{m \in \mathbb{N}_{0}} \gamma_{m}
$$

where $\gamma_{m}=\{s \in \mathbb{C}:|s|=1\}$ for all $m \in \mathbb{N}_{0}$. By the Tikhonov theorem, with the product topology and pointwise multiplication, the torus $\Omega$ is a compact topological Abelian group. Therefore, $\Omega^{r}=\Omega_{1} \times \cdots \times \Omega_{r}$, where $\Omega_{j}=\Omega$ for all $j=1, \ldots, r$, again is a compact topological Abelian group. Thus, on $\left(\Omega^{r}, \mathcal{B}\left(\Omega^{r}\right)\right.$ ), the probability Haar measure $m_{H}$ can be defined, and we have the probability space $\left(\Omega^{r}, \mathcal{B}\left(\Omega^{r}\right), m_{H}\right)$. Denote by $\omega_{j}(m)$ the $m$ th component of an element $\omega_{j} \in \Omega_{j}, j=1, \ldots, r, m \in \mathbb{N}$. Characters of the group $\Omega^{r}$ are of the form

$$
\prod_{j=1}^{r} \prod_{m \in \mathbb{N}_{0}}^{*} \omega_{j}^{k_{j m}}(m)
$$

where the sign "*" shows that only a finite number of integers $k_{j m}$ are distinct from zero. Therefore, putting $\underline{k}=\left\{k_{j m}: k_{j m} \in \mathbb{Z}, m \in \mathbb{N}_{0}\right\}, j=, \ldots, r$, we have that the Fourier transform $g\left(\underline{k}_{1}, \ldots, \underline{k}_{r}\right)$ of a probability measure $\mu$ on $\left(\Omega^{r}, \mathcal{B}\left(\Omega^{r}\right)\right)$ is given by

$$
g\left(\underline{k}_{1}, \ldots, \underline{k}_{r}\right)=\int_{\Omega^{r}}\left(\prod_{j=1}^{r} \prod_{m \in \mathbb{N}_{0}}^{*} \omega_{j}^{k_{j m}}(m)\right) \mathrm{d} \mu .
$$


Define two collections

$$
\begin{aligned}
& A(\underline{\alpha}, \underline{h})=\left\{\left(\underline{k}_{1}, \ldots, \underline{k}_{r}\right): \exp \left\{-i \sum_{j=1}^{r} h_{j} \sum_{m \in \mathbb{N}_{0}}^{*} k_{j m} \log \left(m+\alpha_{j}\right)\right\}=1\right\}, \\
& B(\underline{\alpha}, \underline{h})=\left\{\left(\underline{k}_{1}, \ldots, \underline{k}_{r}\right): \exp \left\{-i \sum_{j=1}^{r} h_{j} \sum_{m \in \mathbb{N}_{0}}^{*} k_{j m} \log \left(m+\alpha_{j}\right)\right\} \neq 1\right\} .
\end{aligned}
$$

Let $Q_{\underline{\alpha}, \underline{h}}$ be the probability measure on $\left(\Omega^{r}, \mathcal{B}\left(\Omega^{r}\right)\right)$ having the Fourier transform

$$
g_{\underline{\alpha}, \underline{h}}\left(\underline{k}_{1}, \ldots, \underline{k}_{r}\right)= \begin{cases}1 & \text { if }\left(\underline{k}_{1}, \ldots, \underline{k}_{r}\right) \in A(\underline{\alpha}, \underline{h}), \\ 0 & \text { if }\left(\underline{k}_{1}, \ldots, \underline{k}_{r}\right) \in B(\underline{\alpha}, \underline{h}) .\end{cases}
$$

For $A \in \mathcal{B}\left(\Omega^{r}\right)$, define

$$
\begin{array}{r}
Q_{N, \underline{\alpha}, \underline{h}}(A)=\frac{1}{N+1} \#\left\{0 \leqslant k \leqslant N:\left(\left(\left(m+\alpha_{1}\right)^{-i k h_{1}}: m \in \mathbb{N}_{0}\right), \ldots,\right.\right. \\
\left.\left.\left(\left(m+\alpha_{r}\right)^{-i k h_{r}}: m \in \mathbb{N}_{0}\right)\right) \in A\right\} .
\end{array}
$$

Lemma 1. $Q_{N, \underline{\alpha}, \underline{h}}$ converges weakly to the measure $Q_{\underline{\alpha}, \underline{h}}$ as $N \rightarrow \infty$.

Proof. In view of $(2.1)$, the Fourier transform $g_{N, \underline{\alpha}, \underline{h}}\left(\underline{k}_{1}, \ldots, \underline{k}_{r}\right)$ of $Q_{N, \underline{\alpha}, \underline{h}}$ is given by

$$
\begin{aligned}
& g_{N, \underline{\alpha}, \underline{h}}\left(\underline{k}_{1}, \ldots, \underline{k}_{r}\right) \\
& =\int_{\Omega^{r}}\left(\prod_{j=1}^{r} \prod_{m \in \mathbb{N}_{0}}^{*} \omega_{j}^{k_{j m}}(m)\right) \mathrm{d} Q_{N, \underline{\alpha}, \underline{h}}=\frac{1}{N+1} \sum_{k=0}^{N} \prod_{j=1}^{r} \prod_{m \in \mathbb{N}_{0}}^{*}\left(m+\alpha_{j}\right)^{-i k h_{j} k_{j m}} \\
& =\frac{1}{N+1} \sum_{k=0}^{N} \exp \left\{-i k \sum_{j=1}^{r} h_{j} \sum_{m \in \mathbb{N}_{0}}^{*} k_{j m} \log \left(m+\alpha_{j}\right)\right\} .
\end{aligned}
$$

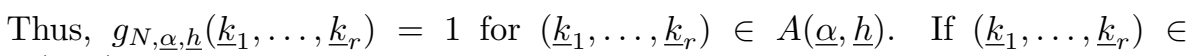
$B(\underline{\alpha}, \underline{h})$, then by the sum formula of geometric progression, we have

$$
\begin{aligned}
g_{N, \underline{\alpha}, \underline{h}} & \left(\underline{k}_{1}, \ldots, \underline{k}_{r}\right) \\
= & \frac{1-\exp \left\{-i(N+1) \sum_{j=1}^{r} h_{j} \sum_{m \in \mathbb{N}_{0}}^{*} k_{j m} \log \left(m+\alpha_{j}\right)\right\}}{(N+1)\left(1-\exp \left\{-i \sum_{j=1}^{r} h_{j} \sum_{m \in \mathbb{N}_{0}}^{*} k_{j m} \log \left(m+\alpha_{j}\right)\right\}\right)} .
\end{aligned}
$$

Therefore,

$$
\lim _{N \rightarrow \infty} g_{N, \underline{\alpha}, \underline{h}}\left(\underline{k}_{1}, \ldots, \underline{k}_{r}\right)= \begin{cases}1 & \text { if }\left(\underline{k}_{1}, \ldots, \underline{k}_{r}\right) \in A(\underline{\alpha}, \underline{h}), \\ 0 & \text { if }\left(\underline{k}_{1}, \ldots, \underline{k}_{r}\right) \in B(\underline{\alpha}, \underline{h}),\end{cases}
$$

This together with a continuity theorem for probability measures on compact groups proves the lemma. 
Now, let $\theta>1 / 2$ be a fixed number, and, for $m \in \mathbb{N}_{0}, n \in \mathbb{N}$,

$$
v_{n}\left(m, \alpha_{j}\right)=\exp \left\{-\left(\frac{m+\alpha_{j}}{n+\alpha_{j}}\right)^{\theta}\right\}, \quad j=1, \ldots, r .
$$

Define $\underline{\zeta}_{n}(s, \underline{\alpha})=\left(\zeta_{n}\left(s, \alpha_{1}\right), \ldots, \zeta_{n}\left(s, \alpha_{r}\right)\right)$, where

$$
\zeta_{n}\left(s, \alpha_{j}\right)=\sum_{m=0}^{\infty} \frac{v_{n}\left(m, \alpha_{j}\right)}{\left(m+\alpha_{j}\right)^{s}}, \quad j=1, \ldots, r .
$$

In view of the definition $v_{n}\left(m, \alpha_{j}\right)$, the latter Dirichlet series are absolutely convergent for $\sigma>1 / 2$. For $A \in \mathcal{B}\left(H^{r}(D)\right)$, define

$$
V_{N, n, \underline{\alpha}, \underline{h}}(A)=\frac{1}{N+1} \#\left\{0 \leqslant k \leqslant N: \underline{\zeta}_{n}(s+i k \underline{h}, \underline{\alpha}) \in A\right\} .
$$

To obtain the weak convergence for $V_{N, n, \underline{\alpha}, \underline{h}}$ as $N \rightarrow \infty$, introduce the mapping $u_{n, \underline{\alpha}}: \Omega^{r} \rightarrow H^{r}(D)$ given by

$$
u_{n, \underline{\alpha}}(\omega)=\underline{\zeta}_{n}(s, \underline{\alpha}, \omega), \quad \omega=\left(\omega_{1}, \ldots, \omega_{r}\right) \in \Omega^{r},
$$

where $\underline{\zeta}_{n}(s, \underline{\alpha}, \omega)=\left(\zeta_{n}\left(s, \alpha_{1}, \omega_{1}\right), \ldots, \zeta_{n}\left(s, \alpha_{r}, \omega_{r}\right)\right)$ with

$$
\zeta_{n}\left(s, \alpha_{j}, \omega_{j}\right)=\sum_{m=0}^{\infty} \frac{\omega_{j}(m) v_{n}\left(m, \alpha_{j}\right)}{\left(m+\alpha_{j}\right)^{s}}, \quad j=1, \ldots, r
$$

Obviously, the latter series also are absolutely convergent for $\sigma>1 / 2$. Therefore, the mapping $u_{n}$ is continuous, hence, it is $\left(\mathcal{B}\left(\Omega^{r}\right), \mathcal{B}\left(H^{r}(D)\right)\right)$-measurable. Thus, the measure $Q_{\underline{\alpha}, \underline{h}}$ defines the unique probability measure $V_{\underline{\alpha}, \underline{h}}$ on $\left(H^{r}(D)\right.$, $\left.\mathcal{B}\left(H^{r}(D)\right)\right)$ by the formula

$$
V_{\underline{\alpha}, \underline{h}}(A)=Q_{\underline{\alpha}, \underline{h}}\left(u_{n, \underline{\alpha}}^{-1} A\right), \quad A \in \mathcal{B}\left(H^{r}(D)\right) .
$$

Moreover, the definitions of $V_{N, n, \underline{\alpha}, \underline{h}}$ and $Q_{N, \underline{\alpha}, \underline{h}}$ imply the equality

$$
V_{N, n, \underline{\alpha}, \underline{h}}(A)=Q_{N, \underline{\alpha}, \underline{h}}\left(u_{n, \underline{\alpha}}^{-1} A\right), \quad A \in \mathcal{B}\left(H^{r}(D)\right) \text {. }
$$

All these remarks together with Lemma 1 and the property of preservation of weak convergence under continuous mappings lead to the following limit lemma.

Lemma 2. $V_{N, n, \underline{\alpha}, \underline{h}}$ converges weakly to $V_{n, \underline{\alpha}, \underline{h}}$ as $N \rightarrow \infty$.

To obtain a limit theorem for $P_{N, \underline{\alpha}, \underline{h}}$, we need the estimation a distance between $\underline{\zeta}_{n}(s, \underline{\alpha})$ and $\underline{\zeta}(s, \underline{\alpha})$. Let $g_{1}, g_{2} \in H(D)$. Recall that

$$
\rho\left(g_{1}, g_{2}\right)=\sum_{l=1}^{\infty} 2^{-l} \frac{\sup _{s \in K_{l}}\left|g_{1}(s)-g_{2}(s)\right|}{1+\sup _{s \in K_{l}}\left|g_{1}(s)-g_{2}(s)\right|},
$$


where $\left\{K_{l}: l \in \mathbb{N}\right\}$ is a certain sequence of compact subsets of the strip $D$, is a metric on $H(D)$ inducing its topology of uniform convergence on compacta. Let $\underline{g}_{1}=\left(g_{11}, \ldots, g_{1 r}\right), \underline{g}_{2}=\left(g_{21}, \ldots, g_{2 r}\right) \in H^{r}(D)$. Then

$$
\underline{\rho}\left(\underline{g}_{1}, \underline{g}_{2}\right)=\max _{1 \leqslant j \leqslant r} \rho\left(g_{1 j}, g_{2 j}\right)
$$

is a metric on $H^{r}(D)$ that induces the product topology.

Let $\theta$ be the same parameter as in definition of $v_{n}\left(m, \alpha_{j}\right)$, and

$$
l_{n}(s, \alpha)=\frac{s}{\theta} \Gamma\left(\frac{s}{\theta}\right)(n+\alpha)^{-s},
$$

where $\Gamma(s)$ is the Euler gamma-function. Then the following integral representation is known [28].

Lemma 3. For $s \in D$,

$$
\zeta(s, \alpha)=\frac{1}{2 \pi i} \int_{\theta-i \infty}^{\theta+i \infty} \zeta(s+z, \alpha) l_{n}(z, \alpha) \frac{\mathrm{d} z}{z} .
$$

We will use some mean square results of discrete type. For the proof of them, the next lemma connecting the continuous and discrete mean squares is useful.

Lemma 4. Suppose that $T, T_{0} \geqslant \delta>0$ are real numbers, $\mathcal{T} \neq \varnothing$ is a finite set lying in the interval $\left[T_{0}+\delta / 2, T_{0}+T-\delta / 2\right]$, and

$$
N_{\delta}(x)=\sum_{t \in \mathcal{T},|t-x|<\delta} 1 \text {. }
$$

Let $S(x)$ be a complex valued function continuous in $\left[T_{0}, T_{0}+T\right]$ and have a continuous derivative in $\left(T_{0}, T_{0}+T\right)$. Then

$\sum_{t \in \mathcal{T}} N_{\delta}^{-1}(t)|S(t)|^{2} \leqslant \frac{1}{\delta} \int_{T_{0}}^{T_{0}+T}|S(x)|^{2} \mathrm{~d} x+\left(\int_{T_{0}}^{T_{0}+T}|S(x)|^{2} \mathrm{~d} x \int_{T_{0}}^{T_{0}+T}\left|S^{\prime}(x)\right|^{2} \mathrm{~d} x\right)^{1 / 2}$.

The lemma is called the Gallagher lemma, its proof is given in [34, Lemma 1.4].

Lemma 5. Suppose that $0<\alpha \leqslant 1,1 / 2<\sigma<1$ and $h>0$ are fixed numbers. Then, for every $t \in \mathbb{R}$,

$$
\sum_{k=0}^{N}|\zeta(\sigma+i k h+i t, \alpha)|^{2} \ll_{\alpha, \sigma, h} N(1+|t|) .
$$

Proof. It is well known that

$$
\int_{0}^{T}|\zeta(\sigma+i t, \alpha)|^{2} \ll_{\alpha, \sigma} T, \quad \int_{0}^{T}\left|\zeta^{\prime}(\sigma+i t, \alpha)\right|^{2} \ll_{\alpha, \sigma} T .
$$

Therefore, an application of Lemma 4 with $\delta=h$ gives the estimate of the lemma.

The next lemma is very important for the proof of weak convergence for $P_{N, \underline{\alpha}, \underline{h}}$. 
Lemma 6. For arbitrary $0<\alpha_{j} \leqslant 1$ and $h_{j}>0, j=1, \ldots, r$,

$$
\lim _{n \rightarrow \infty} \limsup _{N \rightarrow \infty} \frac{1}{N+1} \sum_{k=0}^{N} \underline{\rho}\left(\underline{\zeta}(s+i k \underline{h}, \underline{\alpha}), \underline{\zeta}_{n}(s+i k \underline{h}, \underline{\alpha})\right)=0 .
$$

Proof. The definition of the metric $\rho$ implies that it suffices to show the equality

$$
\lim _{n \rightarrow \infty} \limsup _{N \rightarrow \infty} \frac{1}{N+1} \sum_{k=0}^{N} \rho\left(\zeta(s+i k h, \alpha), \zeta_{n}(s+i k h, \alpha)\right)=0
$$

for arbitrary $0<\alpha \leqslant 1$ and $h>0$. On the other hand, the latter equality is implied by

$$
\lim _{n \rightarrow \infty} \limsup _{N \rightarrow \infty} \frac{1}{N+1} \sum_{k=0}^{N} \sup _{s \in K}\left|\zeta(s+i k h, \alpha)-\zeta_{n}(s+i k h, \alpha)\right|=0
$$

for every compact subset $K \subset D$.

Thus, let $K \subset D$ be an arbitrary compact set. There exists $\varepsilon>0$ such that all points of the set $K$ lie in the strip $\{s \in \mathbb{C}: 1 / 2+2 \varepsilon \leqslant \sigma \leqslant 1-\varepsilon\}$. Let $s=\sigma+i t \in K$, and $\theta_{1}=\sigma-1 / 2-\varepsilon>0$. Then, in view of Lemma 3 and the residue theorem,

$$
\zeta_{n}(s, \alpha)-\zeta(s, \alpha)=\frac{1}{2 \pi i} \int_{-\theta_{1}-i \infty}^{-\theta_{1}+i \infty} \zeta(s+z, \alpha) l_{n}(z, \alpha) \frac{\mathrm{d} z}{z}+R_{n}(s, \alpha),
$$

where

$$
R_{n}(s, \alpha)=\operatorname{Res}_{z=1} \zeta(s+z, \alpha) l_{n}(z, \alpha) \frac{1}{z}=\frac{l_{n}(1-s, \alpha)}{1-s} .
$$

Hence, for $s \in K$,

$$
\begin{aligned}
& \zeta_{n}(s+i k h, \alpha)-\zeta(s+i k h, \alpha) \ll \sup _{s \in K}\left|R_{n}(s+i k h, \alpha)\right| \\
& \quad+\int_{-\infty}^{\infty}|\zeta(1 / 2+\varepsilon+i k h+i \tau, \alpha)| \sup _{s \in K}\left|\frac{l_{n}(1 / 2+\varepsilon-s+i \tau, \alpha)}{1 / 2+\varepsilon-s+i \tau}\right| \mathrm{d} \tau .
\end{aligned}
$$

Therefore,

$$
\frac{1}{N+1} \sum_{k=0}^{N} \sup _{s \in K}\left|\zeta(s+i k h, \alpha)-\zeta_{n}(s+i k h, \alpha)\right| \ll I_{1}+I_{2},
$$

where

$$
I_{1}=\int_{-\infty}^{\infty}\left(\frac{1}{N+1} \sum_{k=0}^{N}\left|\zeta\left(\frac{1}{2}+\varepsilon+i k h+i \tau, \alpha\right)\right|\right) \sup _{s \in K}\left|\frac{l_{n}(1 / 2+\varepsilon-s+i \tau, \alpha)}{1 / 2+\varepsilon-s+i \tau}\right| \mathrm{d} \tau
$$

and

$$
I_{2}=\frac{1}{N+1} \sum_{k=0}^{N} \sup _{s \in K}\left|R_{n}(s+i k h, \alpha)\right|
$$


The crucial role in the estimation of $l_{n}(s, \alpha)$ is played by the gamma-function. It is well known that there exists $c>0$ such that, uniformly in $\sigma_{1} \leqslant \sigma \leqslant \sigma_{2}$,

$$
\Gamma(\sigma+i t) \ll \exp \{-c|t|\} .
$$

This estimate leads, for $\sigma+i t \in K$, to

$$
\begin{aligned}
\frac{l_{n}(1 / 2+\varepsilon-\sigma-i t+i \tau, \alpha)}{1 / 2+\varepsilon-\sigma-i t+i \tau} & \ll \frac{(n+\alpha)^{1 / 2+\varepsilon-\sigma}}{\theta} \exp \{-(c / \theta)|\tau-t|\} \\
& \ll_{\theta, K}(n+\alpha)^{-\varepsilon} \exp \{-(c / \theta)|\tau|\} .
\end{aligned}
$$

Therefore, in view of Lemma 5 ,

$$
\begin{gathered}
I_{1} \ll_{\theta, K}(n+\alpha)^{-\varepsilon} \int_{-\infty}^{\infty}\left(\frac{1}{N} \sum_{k=0}^{N}|\zeta(1 / 2+\varepsilon+i k h+i \tau, \alpha)|^{2}\right)^{1 / 2} \\
\times \exp \{-(c / \theta)|\tau|\} \mathrm{d} \tau \ll_{\theta, K, \varepsilon, h}(n+\alpha)^{-\varepsilon} .
\end{gathered}
$$

By estimate (2.3) again, we find that, for $s \in K$,

$$
\begin{aligned}
\frac{l_{n}(1-s-i k h, \alpha)}{1-s-i k h} & \ll_{\theta}(n+\alpha)^{1-\sigma} \exp \{-(s / \theta)|k h-t|\} \\
& \ll_{\theta, K}(n+\alpha)^{1 / 2-2 \varepsilon} \exp \{-((c h) / \theta) k\} .
\end{aligned}
$$

Therefore,

$$
I_{2} \ll_{\theta, K}(n+\alpha)^{1 / 2-2 \varepsilon} \frac{1}{N} \sum_{k=0}^{N} \exp \{-((c h) / \theta) k\} \ll_{\theta, K, h}(n+\alpha)^{1 / 2-2 \varepsilon} \frac{\log N}{N} .
$$

This, together with (2.4) and (2.2) proves the lemma.

Now, we define the marginal measures of $V_{n, \underline{\alpha}, \underline{h}}$. For $A \in \mathcal{B}\left(\Omega_{j}\right), j=1, \ldots, r$ define

$$
Q_{N, \alpha_{j}, h_{j}}(A)=\frac{1}{N+1} \#\left\{0 \leqslant k \leqslant N:\left(\left(m+\alpha_{j}\right)^{-i k h_{j}}: m \in \mathbb{N}_{0}\right) \in A\right\} .
$$

Then by Lemma 1 of [27], $Q_{N, \alpha_{j}, h_{j}}$ converges weakly to a certain probability measure $Q_{\alpha_{j}, h_{j}}$ on $\left(\Omega_{j}, \mathcal{B}\left(\Omega_{j}\right)\right)$ as $N \rightarrow \infty, j=1, \ldots, r$. Let the mapping $u_{n, \alpha_{j}}: \Omega_{j} \rightarrow H(D)$ be given by $u_{n, \alpha_{j}}\left(\omega_{j}\right)=\zeta_{n}\left(s, \alpha_{j}, \omega_{j}\right)$. Define

$V_{n, \alpha_{j}, h_{j}}(A)=Q_{\alpha_{j}, h_{j}} u_{n, \alpha_{j}}^{-1}(A)=Q_{\alpha_{j}, h_{j}}\left(u_{n, \alpha_{j}}^{-1} A\right), \quad A \in \mathcal{B}(H(D)), j=1, \ldots, r$.

Then in [27, Lemma 4], the following statement has been obtained.

Lemma 7. For all $0<\alpha_{j} \leqslant 1$ and $h_{j}>0, j=1, \ldots, r$, the family of probability measures $\left\{V_{n, \alpha_{j}, h_{j}}: n \in \mathbb{N}\right\}$ is tight, i.e., for every $\varepsilon>0$, there exists a compact set $K_{j}=K_{j}(\varepsilon) \subset H(D)$ such that $V_{n, \alpha_{j}, h_{j}}\left(K_{j}\right)>1-\varepsilon$ for all $n \in \mathbb{N}$.

We apply Lemma 7 for the family of probability measures $\left\{V_{n, \underline{\alpha}, \underline{h}}: n \in \mathbb{N}\right\}$. 
Lemma 8. The family $\left\{V_{n, \underline{\alpha}, \underline{h}}: n \in \mathbb{N}\right\}$ is tight.

Proof. Let $\varepsilon>0$ be an arbitrary number. By Lemma 7 , there exist compact sets $K_{1}, \ldots, K_{r} \subset H(D)$ such that

$$
V_{n, \alpha_{j}, h_{j}}\left(K_{j}\right)>1-\varepsilon / r
$$

for all $n \in \mathbb{N}$. Let $K=K_{1} \times \cdots \times K_{r}$. Then $K$ is a compact set in $H^{r}(D)$. Denoting

$$
\left(H(D) \backslash K_{j}\right)_{r}=(\underbrace{H(D) \times \cdots \times H(D)}_{j-1} \times\left(H(D) \backslash K_{j}\right) \times H(D) \times \cdots \times H(D)),
$$

by $(2.5)$, we have

$V_{n, \underline{\alpha}, \underline{h}}\left(H^{r}(D) \backslash K\right)=V_{n, \underline{\alpha}, \underline{h}}\left(\bigcup_{j=1}^{r}\left(H(D) \backslash K_{j}\right)\right)_{r} \leqslant \sum_{j=1}^{r} V_{n, \alpha_{j}, h_{j}}\left(H(D) \backslash K_{j}\right) \leqslant \varepsilon$

for all $n \in \mathbb{N}$. Thus, $V_{n, \underline{\alpha}, \underline{h}}(K) \geqslant 1-\varepsilon$ for all $n \in \mathbb{N}$.

Now we are in position to prove a limit theorem for $P_{N, \underline{\alpha}, \underline{h}}$.

Theorem 3. On $\left(H^{r}(D), \mathcal{B}\left(H^{r}(D)\right)\right)$, there exists a probability measure $P_{\underline{\alpha}} \underline{h}$ such that $P_{N, \underline{\alpha}, \underline{h}}$ converges weakly to $P_{\underline{\alpha}, \underline{h}}$ as $N \rightarrow \infty$.

Proof. Let $\xi_{N}$ be a random variable defined on a certain probability space with measure $\mu$ and having the distribution

$$
\mu\left\{\xi_{N}=k\right\}=1 /(N+1), \quad k=0,1, \ldots, N .
$$

On the mentioned probability space, define the $H^{r}(D)$-valued random elements

$$
X_{N, n, \underline{\alpha}, \underline{h}}=X_{N, n, \underline{\alpha}, \underline{h}}(s)=\zeta_{n}\left(s+i \xi_{N} \underline{h}, \underline{\alpha}\right), X_{N, \underline{\alpha}, \underline{h}}=X_{N, \underline{\alpha}, \underline{h}}(s)=\zeta\left(s+i \xi_{N} \underline{h}, \underline{\alpha}\right) .
$$

Moreover, let $Y_{n, \underline{\alpha}, \underline{h}}$ be the $H^{r}(D)$-valued random element having the distribution $V_{n, \underline{\alpha}, \underline{h}}$. Then, in view of Lemma 2 ,

$$
X_{N, n, \underline{\alpha}, \underline{h}} \underset{N \rightarrow \infty}{\stackrel{\mathcal{D}}{\longrightarrow}} Y_{n, \underline{\alpha}, \underline{h}},
$$

where $\stackrel{\mathcal{D}}{\rightarrow}$ means the convergence in distribution.

By the Prokhorov theorem, see, for example, [4], every tight family of probability measures is relatively compact. Thus, in view of Lemma 8 , the family $\left\{V_{n, \underline{\alpha}, \underline{h}}\right\}$ is relatively compact. Therefore, there exists a subsequence $\left\{V_{n_{l}, \underline{\alpha}, \underline{h}}\right\}$ weakly convergent to a certain probability measure $P_{\underline{\alpha}, \underline{h}}$ as $l \rightarrow \infty$. Hence,

$$
Y_{n_{l}, \underline{\alpha}, \underline{h}} \underset{l \rightarrow \infty}{\stackrel{\mathcal{D}}{\longrightarrow}} P_{\underline{\alpha}, \underline{h}} .
$$


Moreover, Lemma 6 implies that, for every $\varepsilon>0$,

$$
\begin{aligned}
\lim _{n \rightarrow \infty} & \limsup _{N \rightarrow \infty} \mu\left\{\underline{\rho}\left(X_{N, \underline{\alpha}, \underline{h}}, X_{N, n, \underline{\alpha}, \underline{h}}\right) \geqslant \varepsilon\right\} \\
& \leqslant \lim _{n \rightarrow \infty} \limsup _{N \rightarrow \infty} \frac{1}{\varepsilon(N+1)} \sum_{k=0}^{N} \underline{\rho}\left(\underline{\zeta}(s+i k \underline{h}, \underline{\alpha}), \underline{\zeta}_{n}(s+i k \underline{h}, \underline{\alpha})\right)=0 .
\end{aligned}
$$

This, (2.6) and (2.7) together with Theorem 4.2 of [4] show that

$$
X_{N, \underline{\alpha}, \underline{h}} \underset{N \rightarrow \infty}{\stackrel{\mathcal{D}}{\longrightarrow}} P_{\underline{\alpha}, \underline{h}} .
$$

Since the latter relation is equivalent to weak convergence of $P_{N, \underline{\alpha}, \underline{h}}$ to $P_{\underline{\alpha}, \underline{h}}$ as $N \rightarrow \infty$, the theorem is proved.

\section{Proof of approximation}

Denote by $F_{\underline{\alpha}, \underline{h}}$ the support of the limit measure $P_{\underline{\alpha}, \underline{h}}$ in Theorem 3. Thus $F_{\underline{\alpha}, \underline{h}} \subset H^{r}(D)$ is a minimal closed set such that $P_{\underline{\alpha}, \underline{h}}\left(\bar{F}_{\underline{\alpha}, \underline{h}}\right)=1$. The set $F_{\underline{\alpha}, \underline{h}}$ consists of all elements $g \in H^{r}(D)$ such that, for every open neighbourhood $G$ of $\underline{g}$, the equality $P_{\underline{\alpha}, \underline{h}}(\bar{G})>1$ is satisfied. Obviously, $F_{\underline{\alpha}, \underline{h}} \neq \varnothing$.

Proof. (Proof of Theorem 2).

1. Let $\left(f_{1}(s), \ldots, f_{r}(s)\right) \in F_{\underline{\alpha}, \underline{h}}$. Define the set

$$
G_{\varepsilon}=\left\{\left(g_{1}, \ldots, g_{r}\right) \in H^{r}(D): \sup _{1 \leqslant j \leqslant r} \sup _{s \in K_{j}}\left|g_{j}(s)-f_{j}(s)\right|<\varepsilon\right\} .
$$

Then $G_{\varepsilon}$ is an open neighbourhood of an element of the support of the measure $P_{\underline{\alpha}, \underline{h}}$, therefore $P_{\underline{\alpha}, \underline{h}}\left(G_{\varepsilon}\right)>0$. Hence, by Theorem 3 and equivalent of weak convergence of probability measures in terms of open sets,

$$
\liminf _{N \rightarrow \infty} P_{N, \underline{\alpha}, \underline{h}}\left(G_{\varepsilon}\right) \geqslant P_{\underline{\alpha}, \underline{h}}\left(G_{\varepsilon}\right)>0 .
$$

This, the definitions of $P_{N, \underline{\alpha}, \underline{h}}$ and $G_{\varepsilon}$ prove the first assertion of the theorem.

2. The boundary of the set $G_{\varepsilon}$ lies in the set

$$
\left\{\left(g_{1}, \ldots, g_{r}\right) \in H^{r}(D): \sup _{1 \leqslant j \leqslant r} \sup _{s \in K_{j}}\left|g_{j}(s)-f_{j}(s)\right|=\varepsilon\right\} .
$$

Therefore, these boundaries do not intersect for different $\varepsilon$. Hence, the set $G_{\varepsilon}$ is a continuity set of the measure $P_{\underline{\alpha}, \underline{h}}$ for all but at most countably many $\varepsilon>0$. Therefore, Theorem 3 together with equivalent of weak convergence of probability measures in terms of continuity sets implies that

$$
\lim _{N \rightarrow \infty} P_{N, \underline{\alpha}, \underline{h}}\left(G_{\varepsilon}\right)=P_{\underline{\alpha}, \underline{h}}\left(G_{\varepsilon}\right)>0
$$

for all but at most countably many $\varepsilon>0$, and the second assertion of the theorem is proved. 


\section{References}

[1] B. Bagchi. The Statistical Behaviuor and Universality Properties of the Riemann Zeta-Function and Other Allied Dirichlet Series. PhD Thesis, Indian Statistical Institute, Calcutta, 1981.

[2] A. Balčiūnas, A. Dubickas and A. Laurinčikas. On the Hurwitz zeta-function with algebraic irrational parameter. Math. Notes, 105(1-2):173-179, 2019. https://doi.org/10.1134/S0001434619010218.

[3] A. Balčiūnas, V. Garbaliauskienè, J. Karaliūnaitè, R. Macaitienè, J. Petuškinaitè and A. Rimkevičienè. Joint discrete approximation of a pair of analytic functions by periodic zeta-functions. Math. Modell. Analysis, 25(1):71-87, 2020. https://doi.org/10.3846/mma.2020.10450.

[4] P. Billingsley. Convergence of Probability Measures. Willey, New York, 1968.

[5] E. Buivydas and A. Laurinčikas. A discrete version of the Mishou theorem. $R a-$ manujan J., 38(2):331-347, 2015. https://doi.org/10.1007/s11139-014-9631-2.

[6] E. Buivydas and A. Laurinčikas. A generalized joint discrete universality theorem for the Riemann and Hurwitz zeta-functions. Lith. Math. J., 55(2):193-206, 2015. https://doi.org/10.1007/s10986-015-9273-0.

[7] E. Buivydas, A. Laurinčikas, R. Macaitienè and J. Rašytė. Discrete universality theorems for the Hurwitz zeta-function. J. Approx. Th., 183:1-13, 2014. https://doi.org/10.1016/j.jat.2014.03.006.

[8] V. Franckevič, A. Laurinčikas and D. Šiaučiūnas. On joint value distribution of Hurwitz zeta-functions. Chebyshevskii Sb., 19(3):219-230, 2018.

[9] V. Franckevič, A. Laurinčikas and D. Šiaučiūnas. On approximation of analytic functions by periodic Hurwitz zeta-functions. Math. Modell. Analysis, 24(1):2033, 2019. https://doi.org/10.3846/mma.2019.002.

[10] S.M. Gonek. Analytic Properties of Zeta and L-Functions. PhD Thesis, University of Michigan, 1979.

[11] A. Javtokas and A. Laurinčikas. Universality of the periodic Hurwitz zeta-function. Integral Transforms Spec. Funct., 17(10):711-722, 2006. https://doi.org/10.1080/10652460600856484.

[12] R. Kačinskaitè and A. Laurinčikas. The joint distribution of periodic zeta-functions. Studia Sci. Math. Hungar., 48(2):257-279, 2011. https://doi.org/10.1556/sscmath.48.2011.2.1162.

[13] A. Laurinčikas. The joint universality of Hurwitz zeta-functions. Šiauliai Math. Semin., 3 (11):169-187, 2008.

[14] A. Laurinčikas. Joint universality of zeta-functions with

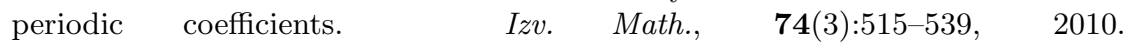
https://doi.org/10.1070/IM2010v074n03ABEH002497.

[15] A. Laurinčikas. A discrete universality theorem for the Hurwitz zeta-function. J. Number Th., 143:232-247, 2014. https://doi.org/10.1016/j.jnt.2014.04.013.

[16] A. Laurinčikas. Distribution modulo 1 and universality of the Hurwitz zeta-function. J. Number Th., 167:294-303, 2016. https://doi.org/10.1016/j.jnt.2016.03.013.

[17] A. Laurinčikas. Universality theorems for zeta-functions with periodic coefficients. Siber. Math. J., $\quad \mathbf{5 7}(2): 330-339,2016$. https://doi.org/10.1134/S0037446616020154. 
[18] A. Laurinčikas. A discrete version of the Mishou theorem. II. Proc. Steklov Inst. Math., 296(1):172-182, 2017. https://doi.org/10.1134/S008154381701014X.

[19] A. Laurinčikas. On discrete universality of the Hurwitz zeta-function. Results Math., 72(1-2):907-917, 2017. https://doi.org/10.1007/s00025-017-0702-8.

[20] A. Laurinčikas. Joint value distribution theorems for the Riemann and Hurwitz zeta-functions. Moscow Math. J., 18(2):349-366, 2018. https://doi.org/10.17323/1609-4514-2018-18-2-349-366.

[21] A. Laurinčikas. "Almost" universality of the Lerch zeta-function. Math. Commun., 24(1):107-118, 2019.

[22] A. Laurinčikas. Joint discrete universality for periodic zeta-functions. Quaest. Math., 42(5):687-699, 2019. https://doi.org/10.2989/16073606.2018.1481891.

[23] A. Laurinčikas. Non-trivial zeros of the Riemann zeta-function and joint universality theorems. J. Math. Anal. Appl., 475(1):385-402, 2019. https://doi.org/10.1016/j.jmaa.2019.02.047.

[24] A. Laurinčikas. On the Mishou theorem with algebraic parameter. Siber. Math. J., 60(6):1075-1082, 2019. https://doi.org/10.1134/S0037446619060144.

[25] A. Laurinčikas. Joint discrete universality for periodic

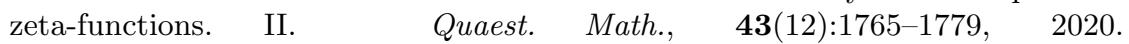
https://doi.org/10.2989/16073606.2019.1654554.

[26] A. Laurinčikas. Zeros of the Riemann zeta-function in the discrete universality of the Hurwitz zeta-function. Studia Sci. Math. Hungar., 57(2):147-164, 2020. https://doi.org/10.1556/012.2020.57.2.1460.

[27] A. Laurinčikas. On the Hurwitz zeta-function with algebraic irrational parameter. Proc. Steklov Inst. Math., http://mi.mathnet.ru/tm4165.

[28] A. Laurinčikas and R. Garunkštis. The Lerch Zeta-Function. Kluwer Academic Publishers, Dordrecht, Boston, London, 2002.

[29] A. Laurinčikas and R. Macaitienè. The discrete universality of the periodic Hurwitz zeta function. Integral Transforms Spec. Funct., 20(9-10):673-686, 2009. https://doi.org/10.1080/10652460902742788.

[30] A. Laurinčikas and R. Macaitienè. Joint approximation of analytic functions by shifts of the Riemann and periodic Hurwitz zeta-functions. Appl. Anal. Discrete Math., 12(2):508-527, 2018. https://doi.org/10.2298/AADM170713016L.

[31] A. Laurinčikas, R. Macaitienè, D. Mochov and D. Šiaučiūnas. Universality of the periodic Hurwitz zeta-function with rational parameter. Sib. Math. J., 59(5):894-900, 2018. https://doi.org/10.1134/S0037446618050130.

[32] R. Macaitienè and D. Šiaučiūnas. Joint universality of Hurwitz zeta-functions and nontrivial zeros of the Riemann zeta-function. Lith. Math. J., 59(1):81-95, 2019. https://doi.org/10.1007/s10986-019-09423-2.

[33] H. Mishou. The joint value distribution of the Rieman zeta-function and Hurwitz zeta-functions. Lith. Math. J., 47(1):32-47, 2007. https://doi.org/10.1007/s10986-007-0003-0.

[34] H.L. Montgomery. Topics in Multiplicative Number Theory. Lecture Notes Math. Vol. 227, Springer-Verlag, Berlin, 1971. https://doi.org/10.1007/BFb0060851.

[35] J. Sander and J. Steuding. Joint universality for sums and products of Dirichlet L-functions. Analysis (Munich), 26(3):295-312, 2006. https://doi.org/10.1524/anly.2006.26.99.295.

[36] S.M. Voronin. Analytic Properties of Generating Function of Arithmetic Objects. Diss. doktor fiz.-matem. nauk, Steklov Math. Inst., Moscow, 1977 (in Russian). 